\title{
Topology optimization of compact wideband coaxial-to-waveguide transitions with minimum-size control
}

\author{
Emadeldeen Hassan $^{1,2}$ (D) . Eddie Wadbro ${ }^{1} \cdot$ Linus Hägg $^{1} \cdot$ Martin Berggren $^{1}$
}

Received: 10 April 2017 / Revised: 8 September 2017 / Accepted: 20 October 2017 / Published online: 15 November 2017

(C) The Author(s) 2017. This article is an open access publication

\begin{abstract}
This paper presents a density-based topology optimization approach to design compact wideband coaxialto-waveguide transitions. The underlying optimization problem shows a strong self penalization towards binary solutions, which entails mesh-dependent designs that generally exhibit poor performance. To address the self penalization issue, we develop a filtering approach that consists of two phases. The first phase aims to relax the self penalization by using a sequence of linear filters. The second phase relies on nonlinear filters and aims to obtain binary solutions and to impose minimum-size control on the final design. We present results for optimizing compact transitions between a 50-Ohm coaxial cable and a standard WR90 waveguide operating in the X-band (8-12 GHz).
\end{abstract}

Keywords Topology optimization · Compact transition . Nonlinear filter $\cdot$ Waveguide $\cdot$ Coaxial cable $\cdot$ Maxwell equations

Emadeldeen Hassan

emad@cs.umu.se

Eddie Wadbro

eddiew@cs.umu.se

Linus Hägg

linush@cs.umu.se

Martin Berggren

martinb@cs.umu.se

1 Department of Computing Science, Umeå University, SE-901 87 Umeå, Sweden

2 Department of Electronics and Electrical Communications, Menoufia University, 32952 Menouf, Egypt

\section{Introduction}

Modern RF and microwave systems design is continuously shifting toward the use of printed circuit and surface mounted technologies to facilitate mass production and achieve compactness. Transitions are key components of microwave circuits and are used to match signals between transmission lines that have different wave impedance, propagating modes, or directions of propagation. A mismatched transition can have a significant impact on the overall system efficiency and can lead to overheating of the device.

Rectangular waveguides are commonly used to feed horn antennas (Balanis 2005, ch 13), as elements in phased array antennas (Pellegrini et al. 2014), or in material characterization (Chang et al. 1997). Meanwhile, for feeding or measurements purposes, coaxial cables are typically used to couple signals into/from waveguides. Coaxial cables support the TEM mode and possess essentially a constant characteristic impedance, whereas rectangular waveguides support TE or TM modes and have a frequency dependent wave impedance (Pozar 2012).

Coaxial-to-rectangular waveguide transitions operating over narrow frequency bands can be designed using electric probes or magnetic loops, whose configuration depends on a few parameters that are easy to determine (Keam and Williamson 1994; Deshpande et al. 1979; Bialkowski et al. 2000). However, wideband transitions typically include complex, bulky 3D structures that can be complicated to mass produce (Yi et al. 2011; Bang and Ahn 2014; Tako et al. 2014). Simeoni et al. (2006) proposed a compact type of coaxial-to-rectangular waveguide transitions that is suitable for mass production by using printed circuit board technology. However, the use of elementary design shapes together with the requirement on compactness make the proposed transitions exhibit narrow frequency band operation. 
Instead of relying on fixed elementary shapes when determining the layout of the printed circuit board, we will here apply the material distribution (also called density-based) technique of topology optimization to design compact wideband coaxial-to-rectangular waveguide transitions.

During the last decade, topology optimization has started to be applied to the design of various electromagnetic components, such as antennas (Nomura et al. 2007; Erentok and Sigmund 2011; Zhou et al. 2010; Aage 2011; Hassan et al. 2014b), metamaterials (Diaz and Sigmund 2010; Otomori et al. 2012), and filters (Kiziltas et al. 2004; Nomura et al. 2013; Aage and Egede Johansen 2017). As opposed to classical topology optimization approaches applied to elastic material, the layout optimization of conducting materials suffers from an ohmic barrier issue. This phenomenon stems from the fact that a material with zero (a dielectric) or infinite (a metal) conductivity exhibits no ohmic losses, in contrast to materials with intermediate conductivities. When topology optimization is applied to maximize transmission or reception, the algorithm will quickly drive each material point to either maximum or minimum conductivity values in order to minimize the losses, if no special action is taken. Moreover, it will be difficult for a continuous optimization algorithm to change a material point from metal to dielectric, or vice versa, because of the barrier of the intermediate conductivity values. Another way of expressing the same issue is to say that the problem is self-penalized toward pure dielectric-metal designs. A naive gradient-based topology optimization implementation will lead to a quick convergence of the algorithm to a lossless design, unfortunately with bad performance (Hassan et al. 2014a). This problem can be addressed by density filtering, a tool originally developed to regularize classical topology optimization problems (Bendsøe and Sigmund 2003). In previous works, we have devised a strategy for self-penalized problems based on filtering together with a continuation approach for a decreasing filter radius. We successfully applied this approach first to the design of metallic antennas (Hassan et al. 2014a, b, 2015a). A similar use of filtering as a strategy to combat the self penalization is also suggested by Aage and Egede Johansen (2017).

In the initial stage of such a strategy, when the filter radius is large, the algorithm operates on a design with large areas of material with intermediate conductivities, and as the filter radius is decreased, intermediate conductivity values are driven closer to the extreme values due to the self penalization. In the end, when the filter radius vanishes, almost all material points contain either a low or a high conductivity material. Note that in this strategy, there is no inherent size control of metal or etched areas, since the filter radius successively vanishes. Nevertheless, in a recent work, we successfully used this strategy to design the layout of metal on a printed circuit board serving as the radiating element in a coaxial-to-waveguide transition (Hassan et al. 2017). In that study, the circuit board was positioned centered and in-line with the extension of the waveguide. This position is electrically favorable, since the electric field for the dominant mode has a maximum in the center of the waveguide, and the devices we obtained indeed exhibit very wide-band operational ranges. However, the components are not compact due to the position of the circuit board.

A much smaller component is accomplished by positioning the circuit board parallel and close to one end of the waveguide (see Fig. 1). This configuration is much more challenging to design, since the device is almost short circuited due to its closeness to the metallic back wall. When we attempted to design such a configuration using the continuation strategy that was successful in the in-line configuration (Hassan et al. 2017), the algorithm's lack of control over feature sizes, both for the metallic and nonmetallic (etched) parts, became apparent; the final design contained pronounced areas of scattered material and holes. Such features can increase the ohmic losses in microwave circuits, especially when appearing at the boundaries of the device (Pozar 2012, §2.7), and they can also complicate the manufacturing. It became thus necessary to improve the algorithm to control the feature size of the metallic and the non-metallic, etched parts. Here we present this improved approach, which allows the design of metal and nonmetal regions with minimum-size control, and show how it has been successfully used to design compact coaxial-towaveguide transitions. This scheme operates in two phases. The first phase aims mainly to counteract the self penalization issue by filtering the design variables using a standard linear density filter combined with a continuation approach over a successively reduced filter size - but only down to a predefined finite size corresponding to the selected feature size. The second phase uses a nonlinear filtering of the design variables, based on a sequence of parameterized harmonic mean filters (Svanberg and Svärd 2013), which in the limit yields binary designs, while at the same time

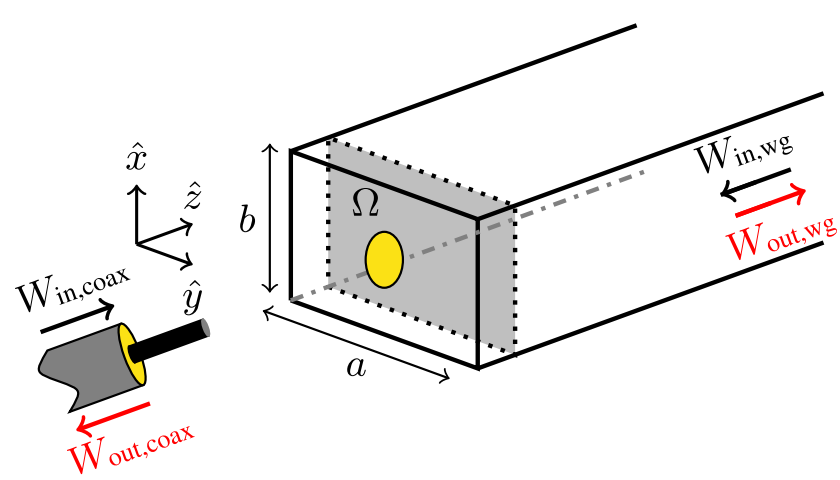

Fig. 1 A 50-Ohm coaxial cable is connected to the rear end of an $a \times b$ rectangular waveguide. The design domain $\Omega$ is used for distributing a conductivity to match the two sides 
maintaining the minimum feature size imposed in the first phase.

\section{Problem statement}

Figure 1 shows the problem setup. A coaxial cable is connected to one end of an $a \times b$ rectangular waveguide. In the electromagnetic community, this setup is commonly called end-launcher transition. We assume that, within the frequency band of interest, the coaxial cable supports only the TEM mode of propagation and has a constant $50 \mathrm{Ohm}$ characteristic impedance. Generally, rectangular waveguides have a frequency dependent wave impedance and support different modes of propagation (Pozar 2012). In this work, the dominant $\mathrm{TE}_{10}$ mode of the rectangular waveguide is considered as the mode of interest. The boundaries of the coaxial cable and the waveguide are assumed to be perfect conductors. Inside the design domain $\Omega$, see Fig. 1, we aim to distribute material with conductivity $\sigma$ to maximize the transmission of signals between the waveguide and the coaxial cable. The domain $\Omega$ is backed by a thin, low-loss dielectric substrate, occupying the volume between $\Omega$ and the shorting wall of the waveguide at $z=0$, to hold the resulting conductivity distribution. The other ends of the coaxial cable and the waveguide are assumed to be matched. That is, the outgoing signals do not reflect back to the analysis domain.

\section{Governing equations and discretization}

The electric field $\mathbf{E}$ and the magnetic field $\mathbf{H}$, inside the waveguide and the coaxial cable, are governed by Maxwell's equations

$$
\begin{array}{r}
\partial_{t} \mu \mathbf{H}+\nabla \times \mathbf{E}=\mathbf{0}, \\
\partial_{t} \in \mathbf{E}+\sigma \mathbf{E}-\nabla \times \mathbf{H}=\mathbf{0},
\end{array}
$$

where $\mu, \epsilon$, and $\sigma$ are the medium permeability, permittivity, and conductivity, respectively. Under the assumption that only the TEM mode is supported by the coaxial cable, the potential difference $V$ and the current $I$ inside the coaxial cable satisfy the following transport equation (Hassan et al. 2014b):

$$
\begin{array}{ll}
\partial_{t}\left(V \pm Z_{\mathrm{c}} I\right) \pm c \partial_{z}\left(V \pm Z_{\mathrm{c}} I\right)=0 & \forall t \text { and } \forall z \\
V+Z_{\mathrm{c}} I=g(t) & \text { at } z=z_{0} .
\end{array}
$$

Here $Z_{\mathrm{c}}$ and $c$ are the characteristic impedance and phase velocity inside the coaxial cable, respectively. The term $\left(V+Z_{\mathrm{c}} I\right)$ indicates a signal traveling in the positive $z$ direction and is used to impose the boundary condition at the coaxial cable end $z_{0}$, and $g(t)$ is chosen to determine the energy spectral density of the imposed signal. The term $\left(V-Z_{c} I\right)$ represents an outgoing signal traveling in the negative $z$-direction. We use this term to estimate the outgoing energy through the coaxial cable end by

$$
W_{\text {out, coax }}=\frac{1}{4 Z_{\mathrm{c}}} \int_{0}^{T}\left(V-Z_{\mathrm{c}} I\right)^{2} \quad d t, \quad \text { at } z_{0}
$$

where $T$ is the total simulation time.

We use the finite-difference time-domain (FDTD) method (Taflove and Hagness 2005) to numerically solve the governing equations (1)-(4). The computational domain is discretized by a uniform cubical Yee grid, where the conductivity is located at the edges. The incoming energy is imposed through the waveguide by the total-field scatteredfield technique. The incoming energy can also be provided by specifying a nonzero function $g(t)$ in boundary condition (4). To simulate the matched ports, the waveguide is terminated by a perfectly matched layer, and (3) together with boundary condition (4) provide perfect absorption of outgoing waves through the coaxial cable end. To control the frequency spectrum of the incoming energy, we use, as a feeding signal, a truncated time-domain sinc pulse modulated to the center of the frequency band of interest (Lathi 1998). The FDTD code is implemented to run on graphics processing units (GPUs) using the parallel computing platform CUDA (https://developer.nvidia.com/what-cuda).

\section{Topology optimization problem}

We first consider the case when the coaxial-to-waveguide system is fed through the waveguide by a time-domain signal that covers the frequency band of interest. For this problem we have, as illustrated in Fig. 1, the following energy balance

$$
W_{\text {in,wg }}=W_{\text {out }, \text { coax }}+W_{\text {out,wg }}+W_{\Omega},
$$

where $W_{\text {in,wg }}$ is the incoming energy associated with the $\mathrm{TE}_{10}$ mode imposed in the waveguide, $W_{\text {out,wg }}$ is the outgoing energy through the waveguide, and $W_{\Omega}$ is ohmic losses inside the design domain $\Omega$. To obtain a good transition between the waveguide and the coaxial cable, a natural objective is to maximize the outgoing energy through the coaxial cable, $W_{\text {out,coax }}$, for the given incoming energy through the waveguide, $W_{\mathrm{in}, \mathrm{wg}}$. According to energy balance (6), the maximization of $W_{\text {out,coax }}$ is equivalent to the minimization of the sum $W_{\text {out,wg }}+W_{\Omega}$.

In our initial experiments for this project, and also in a previous work (Hassan et al. 2015a), we noticed that maximizing the quotient $W_{\text {out,coax }} / W_{\text {out,wg }}$ usually results in optimized designs with favorable performance compared to solely maximizing $W_{\text {out,coax }}$. (Maximizing $W_{\text {out,coax }} / W_{\text {out,wg }}$ effectively corresponds to maximizing the transmission term 
$W_{\text {out,coax }}$ and minimizing the reflection term $W_{\text {out,wg.) So, }}$ to maximize the matching between the waveguide and the coaxial cable, one possibility would be to formulate the conceptual optimization problem

maximize $\log \left(\frac{W_{\text {out,coax }}}{W_{\text {out,wg }}}\right)$

subject to governing equations (1)-(4) and the boundary conditions discussed in the previous section. The performance of microwave devices is typically evaluated using the scattering parameters, which are computed as the logarithm of power ratios. Thus it is natural to use the logarithmic function in the objective function.

As mentioned in the introduction, the waveguide supports different modes of propagation and possesses a frequency dependent wave impedance. In time-domain simulations, these frequency dependencies require complicated treatments regarding the splitting of transient waves and imposing the absorbing boundary condition for the different modes in the rectangular waveguide (Kristensson 1993; Akgun and Tretyakov 2015). To circumvent these complications, we use the fact that the problem under investigation is reciprocal (Pozar 2012). That is, in problem (7), instead of observing $W_{\text {out,wg }}$ given an incoming energy through the waveguide, we observe $W_{\text {out,coax }}$ given an incoming energy through the coaxial cable. We carry out two simulations, one where $W_{\text {in,wg }}$ is nonzero and $g(t)=0$, and one where $W_{\text {in,wg }}=0$ and $g(t)$ is nonzero. Moreover, we rewrite the conceptual problem (7) as

$\operatorname{maximize} \log \left(\frac{W_{\text {out,coax }} \mid W_{\text {in,wg }}}{W_{\text {out,coax }} \mid W_{\text {in, coax }}}\right)$

subject to the governing equations and the boundary conditions discussed in the previous section. In problem (8), $\left.W_{\text {out,coax }}\right|_{W_{\text {in,wg }}}$ and $\left.W_{\text {out,coax }}\right|_{W_{\text {in,coax }}}$ represent the outgoing energy in the coaxial cable given the incoming energy through the waveguide and the coaxial cable, respectively. By this treatment, we are able to use the perfectly matched layer inside the waveguide to simulate a matched port that works for all modes. Moreover, because of transport equation (3), the splitting of a transient wave inside the coaxial cable is much simpler than inside the waveguide. The price for this treatment, however, is that we need to solve the governing equations twice in order to evaluate the objective function.

To solve optimization problem (8), we employ the material distribution topology optimization approach. We use a material indicator vector $\mathbf{p}=\left[p_{1}, p_{2}, \cdots, p_{N}\right]$ to indicate presence, $p_{i}=1$, or absence, $p_{i}=0$, of the conductive material inside the design domain $\Omega$. Our preliminary numerical experiments showed a low sensitivity of the objective function to conductivity values outside the range $\left[\sigma_{\min }=10^{-3} \mathrm{~S} / \mathrm{m}, \sigma_{\max }=10^{5} \mathrm{~S} / \mathrm{m}\right]$. Thus, we map the design vector $\mathbf{p}$, also known as the density vector, to the physical conductivity at the edges of the design grid using

$\boldsymbol{\sigma}=10^{(8 \mathbf{p}-3)} \mathrm{S} / \mathrm{m}$.

To allow gradient-based optimization methods to solve optimization problem (8), the entries of the density vector are allowed to take values between 0 and 1 . The topology optimization problem is to find $\mathbf{p} \in \mathcal{A}=\left\{x \in \mathbb{R}^{N} \mid 0 \leq x_{\mathrm{i}} \leq\right.$ $1 \forall i\}$ that solves problem (8). To compute the objective function gradient, we use an adjoint-field method as detailed by Hassan et al. (2015b). The gradient vector is computed based on the solutions of two adjoint systems corresponding to the two terms $W_{\text {out,coax }} \mid W_{\text {in,wg }}$ and $\left.W_{\text {out,coax }}\right|_{W_{\text {in,coax }}}$ in problem formulation (8).

As mentioned in the introduction, optimization problem (8) is self-penalized toward the lossless cases (that is, toward $\sigma_{\min }$ and $\left.\sigma_{\max }\right)$ due to the energy losses $W_{\Omega}$ associated with the intermediate conductivities, illustrated in Fig. 2. In other words, when gradient-based optimization methods are used to solve this problem, these methods quickly converge to designs consisting of the two extreme values of the design variables, since these values minimize the energy loss. Unfortunately, the quick convergence caused by the self penalization typically results in designs that exhibit bad performance (Hassan et al. 2014a).

\section{Filtering}

Filtering procedures (Sigmund 1994; Bourdin 2001; Bruns and Tortorelli 2001) are among the most popular strategies to achieve mesh-independent designs in topology optimization. Here we consider density filtering methods, where the design variables are filtered and the filtered design variables are mapped to the coefficients that enter the governing equation. A disadvantage of the original linear density filter is that it produces designs with relatively large areas of

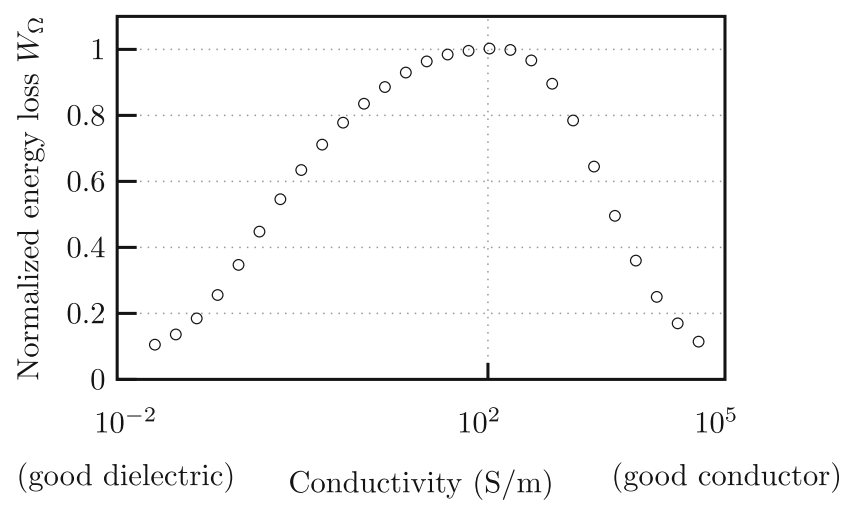

Fig. 2 A typical variation of the energy loss against the values of the design domain conductivity 
intermediate densities. During the last decade there has been an increased interest in nonlinear filtering strategies, aimed at reducing the amount of intermediate densities (Guest et al. 2004, 2011; Sigmund 2007; Svanberg and Svärd 2013; Wadbro and Hägg 2015; Hägg and Wadbro 2017). In addition to providing mesh independent solutions, filtering of the design variables can relieve the strong self penalization discussed in the previous section. To compute the physical conductivity in expression (9), we replace the density vector $\mathbf{p}$ by the filtered density $\tilde{\mathbf{p}}$ obtained by a cascade of $K$ $f W$-mean filters (Wadbro and Hägg 2015),

$\tilde{\mathbf{p}} \stackrel{\text { def }}{=} \mathbf{F}^{(K)} \circ \mathbf{F}^{(K-1)} \circ \cdots \circ \mathbf{F}^{(1)}(\mathbf{p})$,

where

$\mathbf{F}^{(k)}(\mathbf{p})=f_{k}^{-1}\left(\mathbf{W}^{(k)} f_{k}(\mathbf{p})\right) \forall k \in\{1, \cdots, K\}$,

and where the matrix $\mathbf{W}^{(k)}$ determines the weights, shape, and the size of the $k$ th filter; the functions $f_{k}(\cdot)$ and their inverses $f_{k}^{-1}(\cdot)$ are applied elementwise. The filter size is defined by a variable $R$ that defines the radius of a circular neighborhood around each point. In this work, we use equal weights in each neighborhood. The formulation of the generalized $f W$-mean filter allows us to investigate different types of filters based on the choice of the functions $f_{k}(\cdot)$. Here we employ linear as well as nonlinear filters.

\subsection{Linear filter}

By explicitly setting the function $f_{k}(x)=x$ for all $k$, the cascade of $f W$-mean filters reduces to a linear density filter. In this case, the filtering process replaces each design variable $p_{i}$ by a weighted arithmetic mean of the design variables within a neighborhood defined by the weight matrix $\mathbf{W}^{(K)} \mathbf{W}^{(K-1)} \ldots \mathbf{W}^{(1)}$. The linear filter counteracts the self penalization of the optimization problem by producing designs with intermediate values.

To obtain a binary design at the end of the optimization process, we use a continuation approach over the filter radius. In particular, we start by a large filter size $R_{\max }$ and solve a sequence of problems with decreasing filter radii, $R_{n}=\gamma^{n} R_{\max }$ with $\gamma<1$, until the filter radius drops below the spatial discretization step $\Delta$ used in the FDTD grid. In other words, we gradually remove the impact of the filter. Unfortunately, this strategy produces mesh-dependent solutions.

\subsection{Non-linear filter}

To pursue nonlinear filtering of the design variables, we substitute $f_{k}(\cdot)$ in expression (11) by harmonic functions as proposed by Svanberg and Svärd (2013) for elasticity problems. More precisely, we use functions of the form $f_{k}=(x+\alpha)^{-1}$ or $f_{k}=(1-x+\alpha)^{-1}$. The parameter $\alpha>0$ is used to control the nonlinearity of $f_{k}(x)$. When $\alpha$ tends to infinity the harmonic filters approach a linear filter. For small values of $\alpha$ the harmonic filters allow for sharper transitions between regions of different materials than the linear filter. In addition, the harmonic filter operator have bounded derivatives as the nonlinearity parameter $\alpha$ tends to zero. We refer to the study by Svanberg and Svärd (2013) for a comprehensive comparison between the harmonic mean filters and other kinds of filters.

The $f W$-mean filters with the first (second) of the harmonic functions above promotes the values $0(1)$, provided that the input contains 0 (1). The behavior of the two harmonic filters, for small values of $\alpha$, is in this sense similar to the erode and dilate operators in mathematical morphology (Haralick et al. 1987; Heijmans 1995). On a binary design, the dilate operator dilates regions occupied with ones. That is, this operator expands areas occupied by metal. The effect of the erode operator is the opposite in the sense that it erodes regions occupied with ones; that is, it dilates regions occupied with zeros. If we cascade an erode operator followed by a dilate operator, then we get the so-called open operator that removes regions containing ones that are smaller than the neighborhood. Similarly, if we cascade a dilate operator followed by an erode operator, then we get the close operator that removes regions containing zeros that are smaller than the neighborhood.

In the numerical experiments, whenever we use nonlinear filtering, we use an open-close filter; that is, an open filter followed by a close filter. The harmonic openclose filter is thus a cascade of four filters, where $f_{1}(x)=$ $f_{4}(x)=(x+\alpha)^{-1}$ and $f_{2}(x)=f_{3}(x)=f_{1}(1-x)$. We remark that, in general, the open-close filtering strategy does not guarantee minimum size control on both metal and etched regions, as Schevenels and Sigmund (2016) recently reported. Nevertheless, our numerical experiments suggest that the results obtained using an open-close filtering strategy in many cases provide final designs that have smooth boundaries as well as minimum size control on both the metal and etched regions.

\section{Design algorithm}

Figure 3 shows a summary of the proposed optimization algorithm. We start with a uniform distribution of the design variables. The first loop in the design algorithm shows the basic steps to solve a topology optimization problem. In this loop, we filter the design variables, compute the objective function and its gradient, check a convergence criterion, and then, if needed, update the design variables. As convergence criterion, we use the norm of the first-order optimality condition relative to a reference value. More precisely, we start 


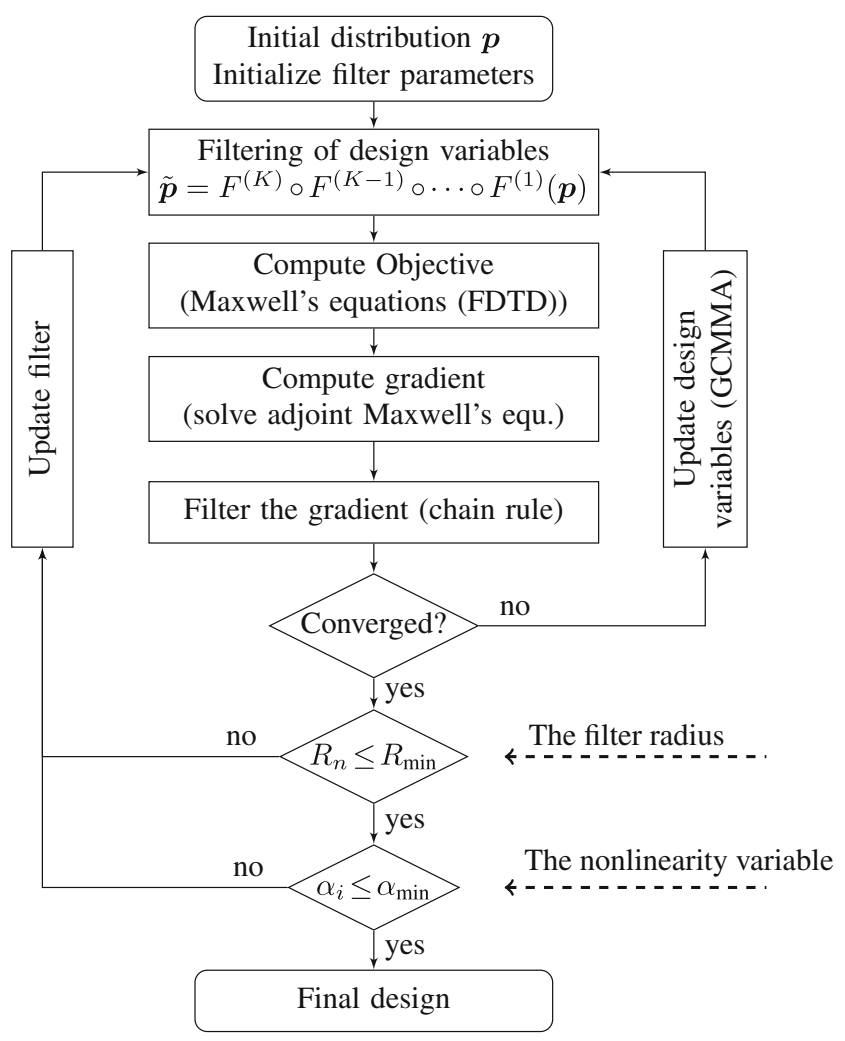

Fig. 3 The optimization algorithm including two continuation levels; one over the filter radius and one over the nonlinearity of the filter

the solution of a problem, record the norm of the first-order optimality condition after 8 iterations, continue the solution of the subproblem until the norm of the first-order optimality condition decreases to $25 \%$ of the recorded value. To update the design variables, we use the globally-convergent method of moving asymptotes (GCMMA) developed by Svanberg (2002).

The second two loops in the design algorithm update the filter parameters. The first of these represents the continuation over the filter radius. In all cases, we update the filter radius using $R_{\mathrm{n}}=\max \left\{(0.75)^{n} \times(10 \Delta), R_{\min }\right\}$, where we set $R_{\min }=\Delta$ when linear filters are used. For the nonlinear filters, we investigate different values for $R_{\min }$. When nonlinear filters are used, the last loop represents the continuation over the nonlinearity variable $\alpha$. We update the nonlinearity variable using $\alpha_{i}=(0.5)^{i} \times \alpha_{\max }$, where $\alpha_{\max }$ is a starting value of $\alpha$ chosen to be large enough. The algorithm terminates when $\alpha_{i}$ drops below $\alpha_{\min }=10^{-6}$. To characterize the amount of grayness in the final design, we use the non-discreteness measure, $M_{\mathrm{nd}}=4 \tilde{\mathbf{p}}^{T}(\mathbf{1}-\tilde{\mathbf{p}}) / N$, suggested by Sigmund (2007), where $N$ is the number of entries in $\tilde{\mathbf{p}}$ and $\mathbf{1}$ is a vector of length $N$ with ones at all entries. Furthermore, in a final post-processing step, we use $1 \mathrm{~S} / \mathrm{m}$ as a threshold conductivity to map values below and above that value to $\sigma_{\min }$ and $\sigma_{\max }$, respectively.

\section{Results and discussion}

We design a transition between a $50 \mathrm{Ohm}$ coaxial cable and a standard WR90 rectangular waveguide. The frequency band $8-12 \mathrm{GHz}$ is considered as the band of interest. The radii of the inner probe and the outer shield of the coaxial cable are $1.27 \mathrm{~mm}$ and $4.45 \mathrm{~mm}$, respectively, and the waveguide dimensions are $22.86 \mathrm{~mm} \times 10.16 \mathrm{~mm}$. The coaxial cable is connected, to the shorting wall at the waveguide end, at a point shifted $2.54 \mathrm{~mm}$ in the negative $x$-direction from the center (see Fig. 1). We use a spatial discretization step $\Delta=0.127 \mathrm{~mm}$ in the FDTD method and a time discretization 0.95 of the Courant step. The design domain $\Omega$, see Fig. 1, located at $z=1.27 \mathrm{~mm}$, is discretized into $180 \times 80$ cells, resulting in 28,540 design variables associated with the interior edges of the grid. The volume between $z=0$ and $z=1.27 \mathrm{~mm}$ is filled by a low-loss RT/Duroid $5880 \mathrm{LZ}$ substrate $\left(\epsilon_{\mathrm{r}}=1.96\right.$ and $\tan \delta=0.002$ at $\left.10 \mathrm{GHz}\right)$ to hold the design. For all design problems in this work, we start with a uniform initial distribution of the design variables, $p_{\mathrm{i}}=0.6$, which corresponds to conductivity values near the peak in Fig. 2. This choice reduces the risk of biasing the design towards any of the two lossless cases (that is, the good dielectric and the good conductor).

\subsection{Linear filter}

In this section, we present the results for designing the transition using a linear filter combined with a continuation approach. First, we set the functions $f_{k}(x)=x$ in expression (11), and use a cascade of four linear filters (that is, $K=4$ in expression (10)). The reason to use the four cascaded linear filters is to obtain a filtering effect, especially in the beginning of the optimization, comparable to the one used in the next section, for the nonlinear filter. Although each filter matrix $\mathbf{W}^{(k)}$ uses constant weights over a circular neighborhood of radius $R$, the cascade of the $f W$-mean filters acts as a linear filter with weighting matrix $\mathbf{W}^{4}$ (filter size 4R) (Hägg and Wadbro 2017).

Figure 4 shows the progress of the objective function together with some snapshots showing the development of the filtered design. We include in the same figure the change in the level of non-discreteness of the filtered design. The objective function increases monotonically with distinct jumps at the beginning of each subproblem, when the filter radius decreases. At the beginning of the optimization, the design contains large amounts of intermediate values ( $\left.M_{\mathrm{nd}}=44 \%\right)$, and the boundaries are blurred, which is expected because of the large filter size. At the end of the optimization process, the design has crisp boundaries and $M_{\text {nd }}$ has decreased to $1.6 \%$.

Figure 5a shows the final conductivity distribution obtained by the optimization algorithm after 111 iterations. 


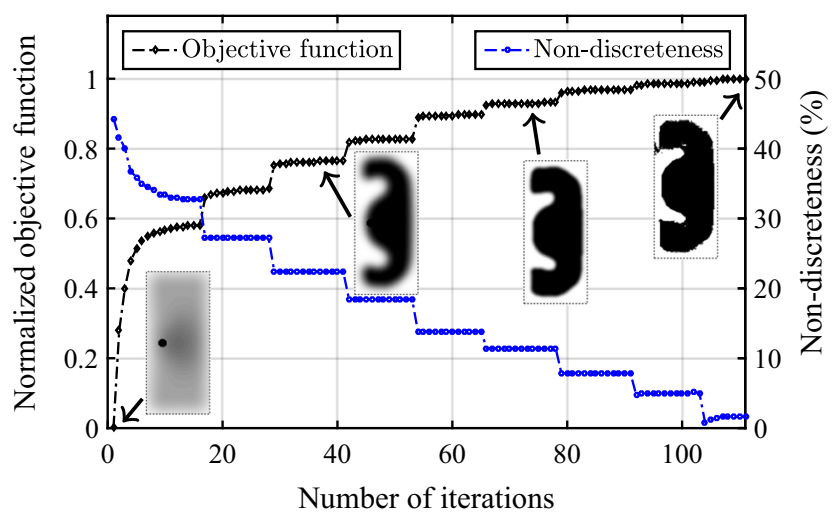

Fig. 4 The iteration history of the objective function and the nondiscreteness level together with some samples of the filtered design. Black color indicates a good conductor and white color indicate a good dielectric

We note that the final design contains small metallic parts as well as small holes inside the metallic block. These small features typically appear in the design when the filter radius is reduced to small values, which indicates that the solution to the optimization problem is mesh dependent. (As we use finer grids, smaller features will likely appear in the final design.) Based on numerical investigations, these small metallic parts and holes have a minor impact on the performance of the transition. This conclusion can also be inferred from the development of the objective function in Fig. 4; there is little variation in the objective function near the end, where the small features start to appear. In practice, however, these small features may complicate the manufacturing process and raise the ohmic losses inside the transition, as mentioned in the introduction. Figure $5 \mathrm{~b}$ shows the amplitudes of the reflection coefficient $\left|S_{11}\right|$ and the coupling coefficient $\left|S_{21}\right|$ of the transition. The performance of the transition is computed by our FDTD code and cross-verified with the commercial CST Microwave Studio package (https://www.cst.com/). Overall, there is a good match between the two simulations; the slight frequency shift can be accounted for by the differences in geometry description between the two methods. The optimized transition has a reflection coefficient, $\left|S_{11}\right|$, below $-8 \mathrm{~dB}$ and coupling coefficient, $\left|S_{21}\right|$, above $-1 \mathrm{~dB}$ over the frequency band $8.5-12 \mathrm{GHz}$, which essentially covers the frequency band of interest marked by the vertically dashed lines in the plot. The simulation results of the transition show that there is a resonance around $11.5 \mathrm{GHz}$, which also appears, unfortunately, in many of the subsequent optimization results. We emphasize that the formulation of the objective function as the integral of the outgoing energy in time-domain makes it difficult to target a specific single frequency. We believe that this resonance is promoted by some geometrical features in the problem setup and leave this issue for future investigations.

We note that the final designs may be asymmetric with respect to the symmetry plane $y=a / 2$ (see Fig. 1). Although the problem setup is symmetric, an optimal design need not be symmetric. In fact, imposing symmetry would restrict the design space, which potentially leads to designs with poorer performance. Here, the asymmetry stems from the use of finite precision arithmetic; although the initial design conductivity is symmetric, the numerically computed fields will generally not be perfectly symmetric. Given asymmetric design conductivities, the optimization algorithm may drive the design back to the symmetric case or away from it depending on the design sensitivities.

To further improve the performance of the transition, we use an additional design layer inside the waveguide at the plane $z=2.54 \mathrm{~mm}$ to distribute the conductivity. The new design domain consists of two layers, one layer at $z=1.27$ $\mathrm{mm}$ and the second layer at $z=2.54 \mathrm{~mm}$, with a low-loss RT/Duroid 5880 LZ substrate filling the volume between
Fig. 5 a The final conductivity distribution over the design domain when a linear filter combined with a continuation over the filter radius is used. $\mathbf{b}$ The scattering parameters of the transition

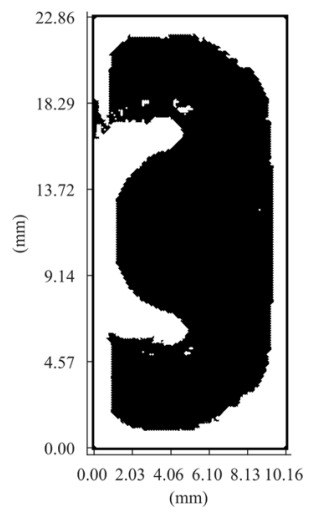

(a)

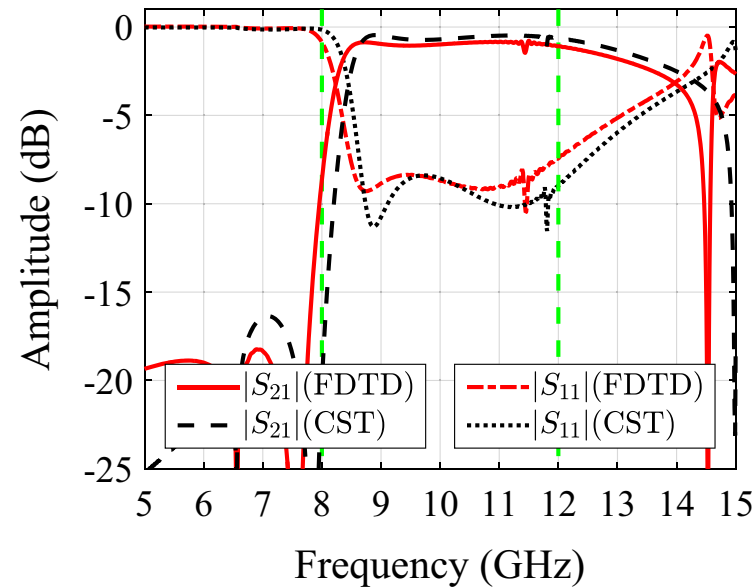

(b) 


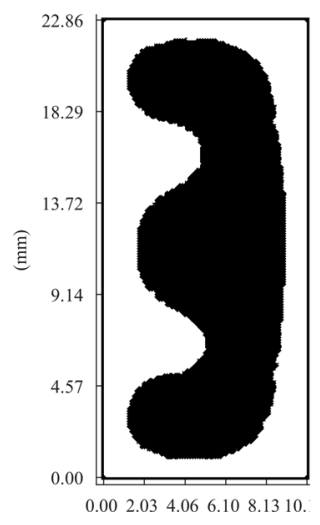

(a)

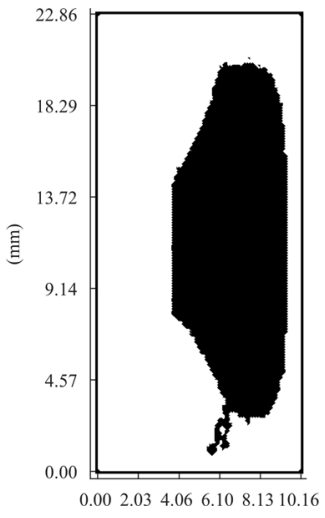

(b)

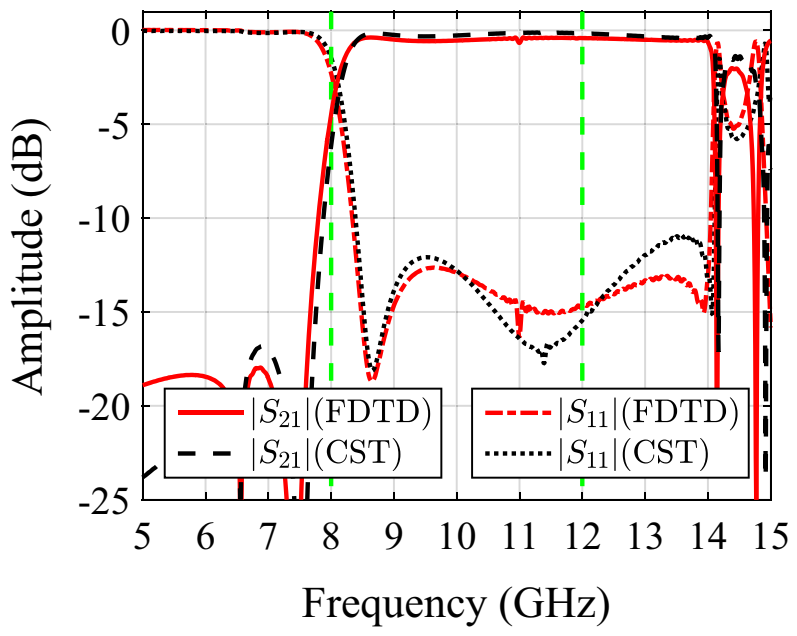

(c)

Fig. 6 Results of optimizing the two-layer design using the linear filtering approach. a First layer conductivity distribution, $z=1.27 \mathrm{~mm}$. $\mathbf{b}$ Second layer conductivity distribution, $z=2.54 \mathrm{~mm}$. $\mathbf{c}$ The scattering parameters of the transition

$z=0 \mathrm{~mm}$ and $z=2.54 \mathrm{~mm}$. The new design problem has 57,080 design variables and is solved using the same settings as the one-layer design case. Figure $6 a$ and $b$ show the final design obtained after 92 iterations. We note the appearance of small metallic parts in the second layer at $z=2.54$ $\mathrm{mm}$. These small features, as mentioned for the one-layer design, appear when the filter size diminishes, since the solution of the optimization problem becomes mesh dependent. The non-discreteness level of the final design is $M_{\mathrm{nd}}=$ $0.3 \%$. Figure $6 \mathrm{c}$ shows the improvement in the performance of the two-layer design compared to Fig. 5b. The $\left|S_{11}\right|$ curve has values below $-12.5 \mathrm{~dB}$ over the frequency band 8.4-12 $\mathrm{GHz}$ with a corresponding $\left|S_{21}\right|$ above $-0.5 \mathrm{~dB}$, excluding the resonance around $11 \mathrm{GHz}$.

To conclude, the approach used in this section has the drawback that small features can appear in the final designs, as shown in Figs. 5a and 6b. These features appear as a consequence of reducing the filter radius during the continuation approach. These small features can increase the ohmic losses (Pozar 2012, §2.7) and cause manufacturing problems.

\subsection{Nonlinear filters}

In this section, we present the results when using the proposed two-phase filtering approach. We use a cascade of four $f W$-mean filters to implement the open-close filter discussed in Section 5. In a one dimensional test problem, we noticed only small differences between filtered designs obtained by using values of $\alpha>8$ in the harmonic openclose ( $f W$-mean) filter and the case when a linear filter is used (that is, when $f_{k}(x)=x$ for all $k$ ). Therefore, in the first phase of the filtering process, Phase I, we fix the nonlinearity variable $\alpha$ to $\alpha_{\max }=8$, and we follow a continuation approach over the filter radius, as in the case of the linear filter. That is, we solve a sequence of problems, for which the the filter radius is updated using $R_{n}=(0.75)^{n} \times(10 \Delta)$. Here, the filter radius is updated until the radius reaches a specified value $R_{\min } \in\{3 \Delta, 5 \Delta, 7 \Delta\}$. In the second phase of the filtering process, Phase II, we fix the filter radius to $R_{\min }$ and start a continuation approach over the nonlinearity variable $\alpha$ aiming for binary solutions. In Phase II, we update the nonlinearity variable using $\alpha_{i}=(0.5)^{i} \times(8)$, and the algorithm stops when $\alpha_{i}$ drops below $10^{-6}$. We remark that if the required final filter size $R_{\min }$ is large, then Phase I only has a minor influence, since the harmonic open-close ( $f W$-mean) filter performs similar to a linear filter in the

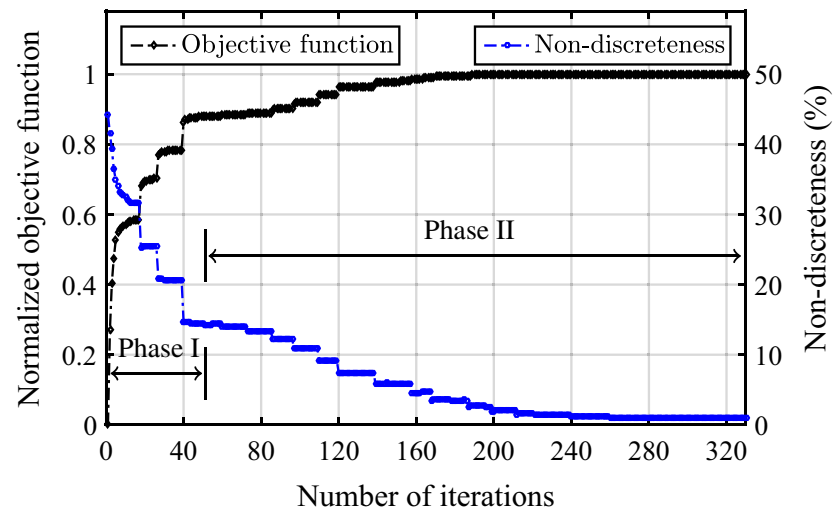

Fig. 7 The objective function and non-discreteness level of the physical design, when we use the two-phase filtering approach to design the one-layer transition 
Fig. 8 a The final conductivity distribution over the one-layer design domain, when we use the two-phase filtering approach with $R_{\min }=3 \Delta$. b The scattering parameters of the transition

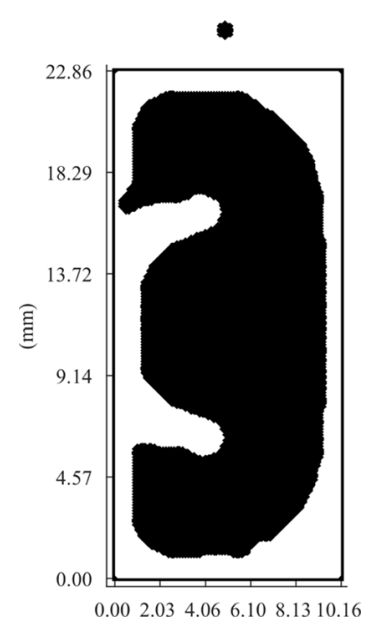

(a)

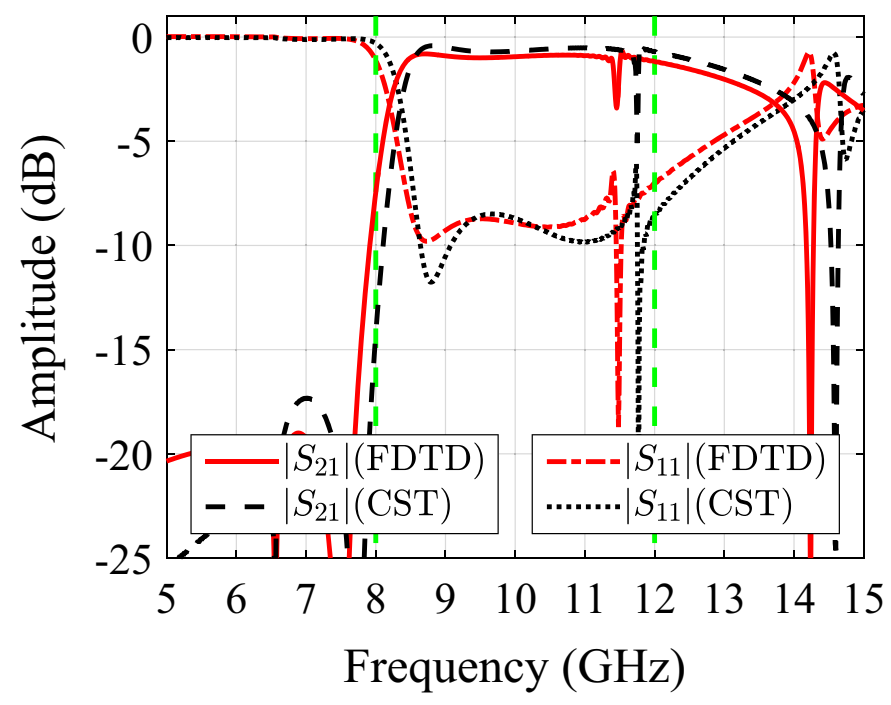

(b) beginning of Phase II. However, in our numerical experiments, we noticed that generally the use of the two-phase filtering approach allows the algorithm to converge to better solutions in terms of the objective function. In particular, Phase I is essential to obtain good results when a small $R_{\text {min }}$ is used.

Figure 7 shows the progress of the objective function and the non-discreteness level of the filtered design for the onelayer design case using the two-phase filtering approach, when $R_{\min }=3 \Delta$ is used. The optimization algorithm converged to the final solution after 330 iterations $(100 \%)$, of which 49 iterations (15\%) are used in Phase I and 281 iterations (85\%) are used in Phase II. By the end of Phase I, the objective function increased to $88 \%$ of the maximum value and the level of non-discreteness decreased to $15 \%$ (from an initial value of 44\%). In Phase II, the change in the objective function is relatively smaller than that in Phase I, nevertheless, it is essential to carefully update the nonlinearity variable $\alpha$ to avoid numerical instabilities in the continuation approach. The design algorithm converged to a final design with $M_{\mathrm{nd}}=1.0 \%$.

Figure 8a shows the final design for the case $R_{\min }=3 \Delta$, with the small circle included above the figure indicating the filter size. We note that, except for the staircasing inherited intrinsically in the FDTD discretiziation, the use of the open-close filter removed both the metallic (black color)
Fig. 9 a The final conductivity distribution over the one-layer design domain, when we use the two-phase filtering approach with $R_{\min }=5 \Delta$. b The scattering parameters of the transition

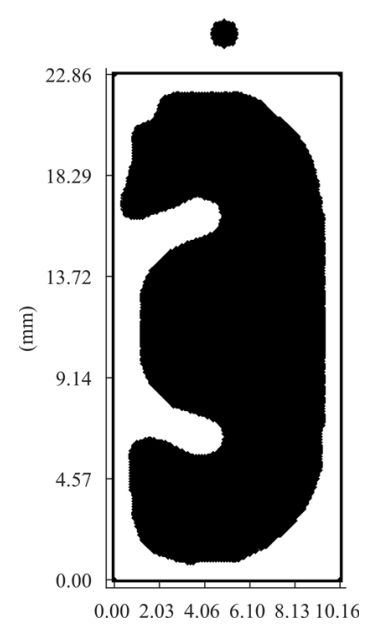

(a)

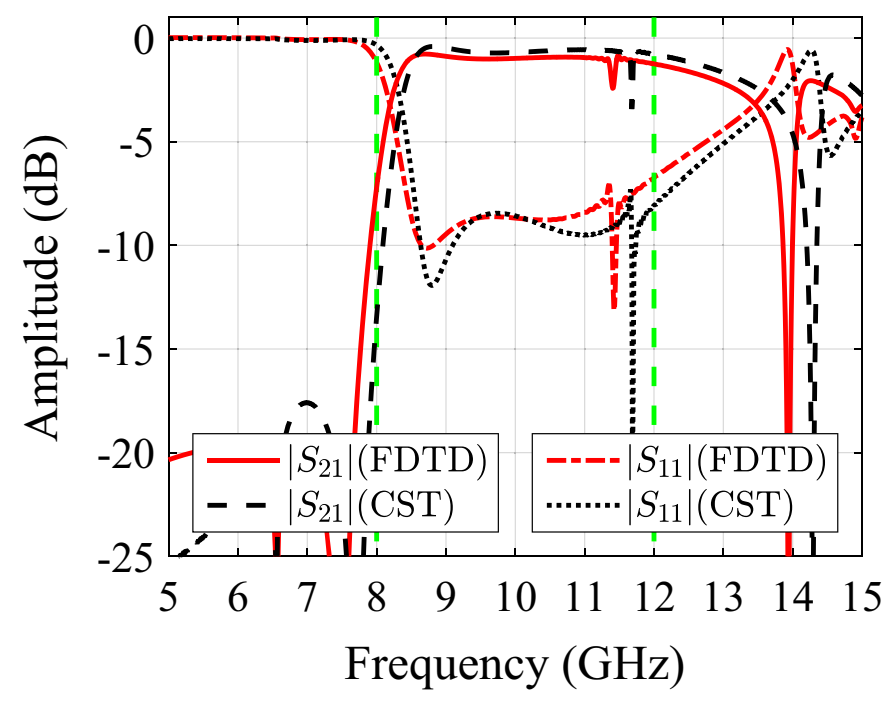

(b) 
Fig. 10 a The final conductivity distribution over the one-layer design domain, when we use the two-phase filtering approach with $R_{\min }=7 \Delta . \mathbf{b}$ The scattering parameters of the transition

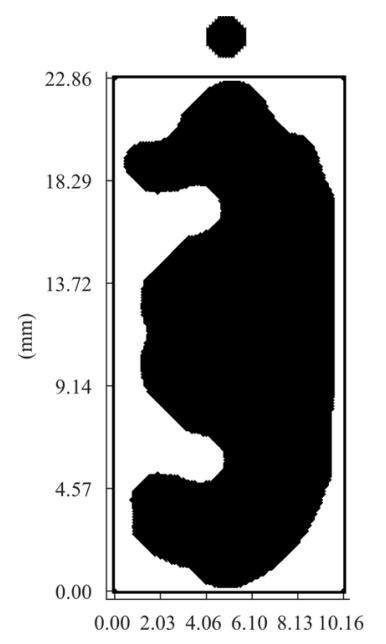

(a)

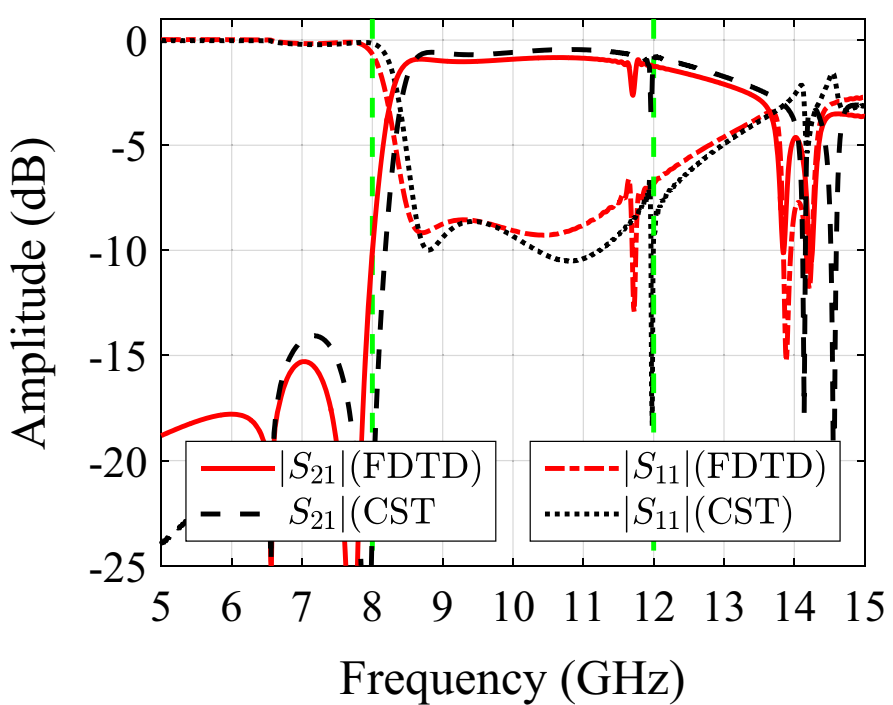

(b) and the dielectric (white color) features smaller than the filter size. The performance of the transition, given in Fig. $8 \mathrm{~b}$, is essentially the same as the one obtained by the linear filter.

To illustrate the effect of the parameter $R_{\min }$, we present two additional designs obtained by the design algorithm for $R_{\min }=5 \Delta$ and $R_{\min }=7 \Delta$, in Figs. 9 and 10 , which were obtained after 336 and 296 iterations with a final $M_{\mathrm{nd}}=$ $0.7 \%$ and $M_{\text {nd }}=1.5 \%$, respectively.

To obtain a quantitative measure of the size control in the final designs, we estimate their minimum sizes by seeking the largest $R$ so that for any edge $e$ in the design there exists a circle $C_{e}$ with radius $R$ that contains $e$ and is inscribed in the design. We estimate the minimum size of the metallic parts as well as the dielectric protrusions in the final designs. The estimated minimum sizes for the metallic parts of the designs in Figs. 8a, 9a, and 10a are $3 \Delta, 6 \Delta$, and $7 \Delta$, respectively. The corresponding dielectric protrusions have estimated minimum sizes $3 \Delta, 5 \Delta$, and $7 \Delta$, respectively. Therefore, we may conclude that the use of the open-close filter imposes minimum size control over the metallic parts as well as the dielectric protrusions, with minor impact on the performance compared to the results obtained with the linear filter.

Similar to the linear filter case, we use the proposed nonlinear filtering to investigate the design of a two-layer

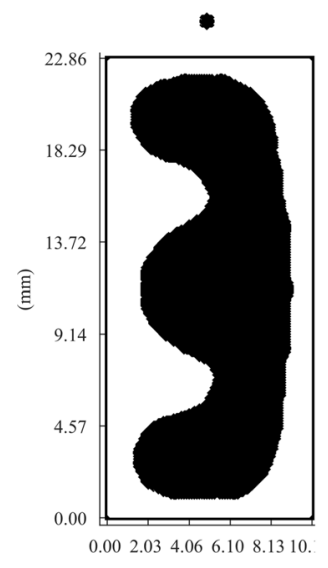

(a)

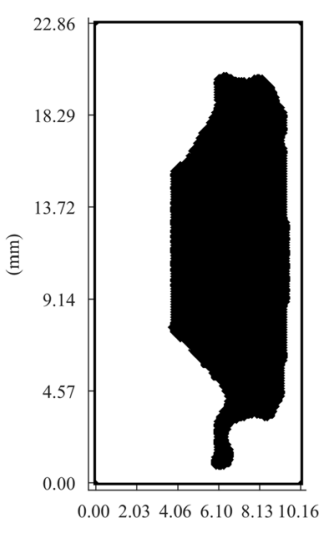

(b)

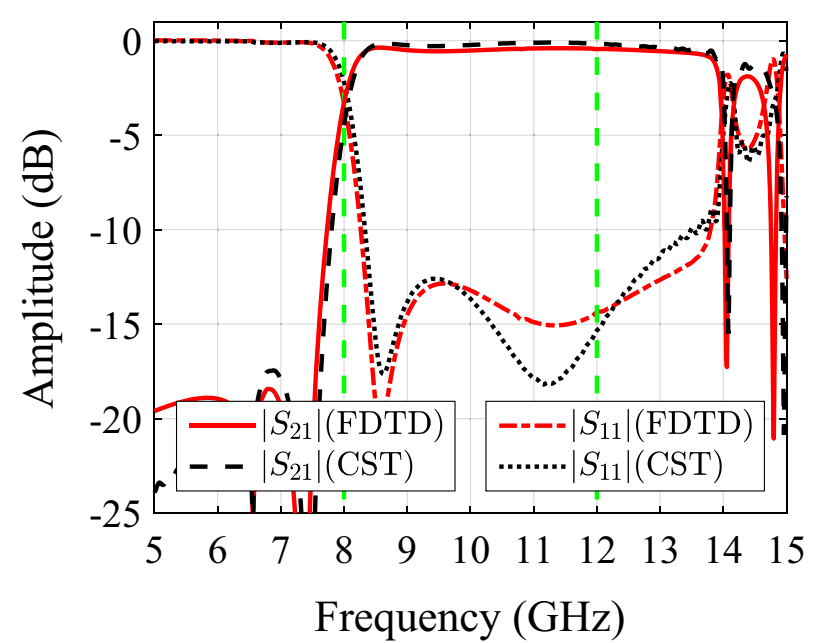

(c)

Fig. 11 Results of optimizing the two-layer design by using the two-phase filtering approach with $R_{\min }=3 \Delta$. a First layer conductivity distribution, $z=1.27 \mathrm{~mm}$. b Second layer conductivity distribution, $z=2.54 \mathrm{~mm}$. $\mathbf{c}$ The scattering parameters of the transition 


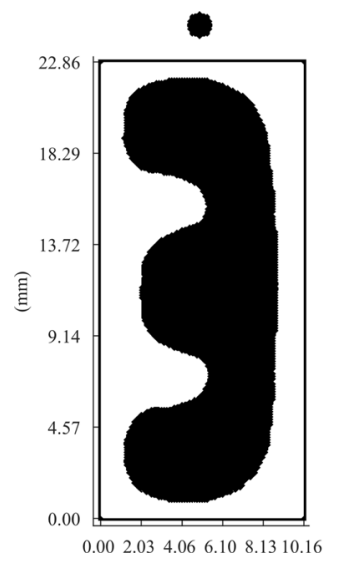

(a)

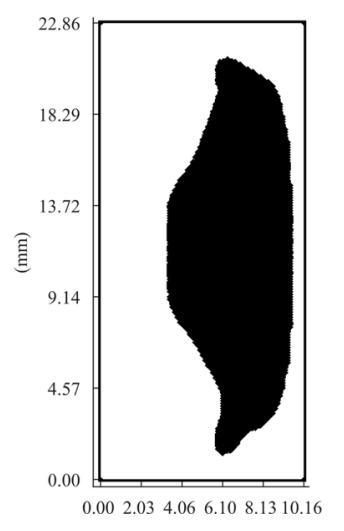

(b)

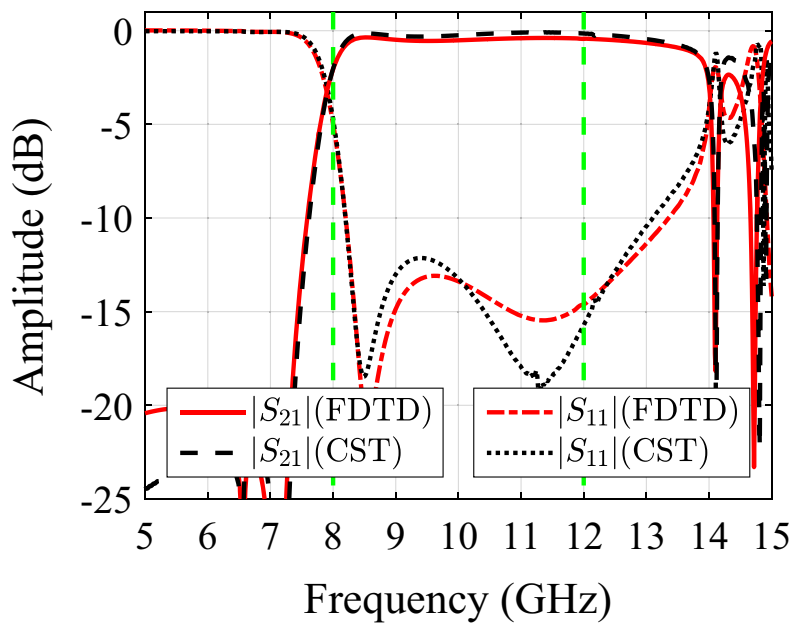

(c)

Fig. 12 Results of optimizing the two-layer design by using the two-phase filtering approach with $R_{\min }=5 \Delta$. a First layer conductivity distribution, $z=1.27 \mathrm{~mm}$. b Second layer conductivity distribution, $z=2.54 \mathrm{~mm}$. $\mathbf{c}$ The scattering parameters of the transition

transition. Figures 11, 12, and 13 show the optimization results obtained by the algorithm when we use $R_{\min }=$ $3 \Delta, R_{\min }=5 \Delta$, and $R_{\min }=7 \Delta$, respectively. These results were obtained by the design algorithm after 334 , 350 , and 316 iterations with final $M_{\mathrm{nd}}$ of $0.3 \%, 0.3 \%$, and $0.9 \%$, respectively. The effect of the filter radius $R_{\min }$ is more prominent on the second layer of the designs in Figs. 11b, 12b, and 13b, where we see that the small features in the final design have a size comparable to the corresponding value of $R_{\min }$. The estimated minimum size of the first layer's metallic parts are $6 \Delta, 11 \Delta$, and $8 \Delta$ for the designs in Figs. 11a, 12a, and 13a, respectively, while the metallic parts in the second layer (Figs. 11b, 12b, and $13 \mathrm{~b}$ ) have estimated minimum sizes $3 \Delta, 5 \Delta$, and $7 \Delta$, respectively. The dielectric protrusions in both layers in Figs. 11,12 , and 13 have estimated minimum size $3 \Delta, 5 \Delta$, and $7 \Delta$, respectively. That is, in all cases and for both the metallic and dielctric parts the estimated minimum sizes are greater than or equal to the employed filter radii. Overall, the use of the open-close filter imposed minimum size control over the features of the designs with minor impact on design performance.

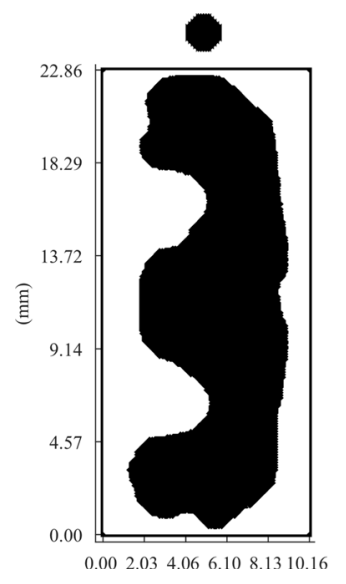

(a)

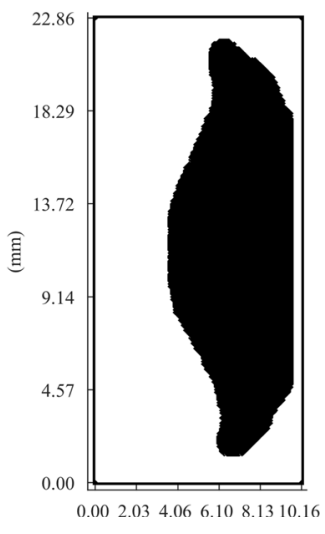

(b)

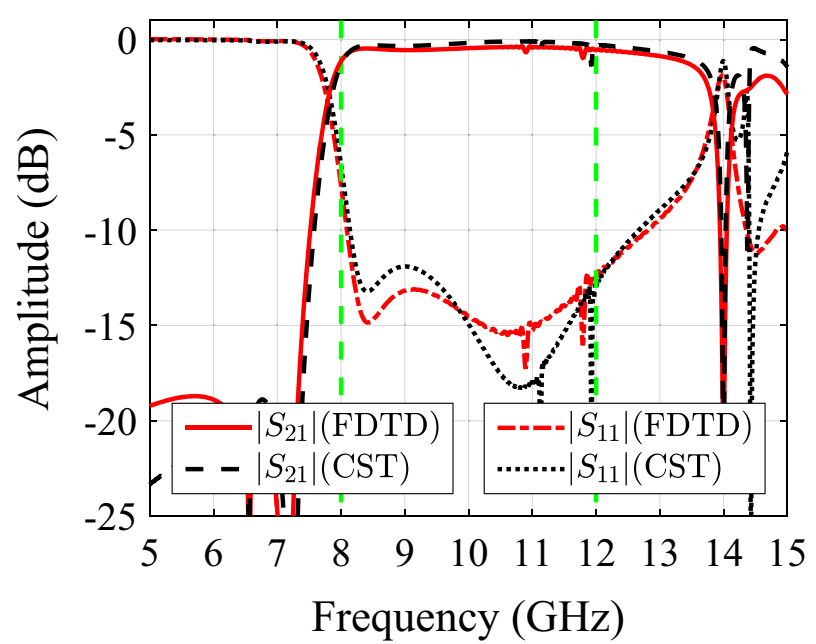

(c)

Fig. 13 Results of optimizing the two-layer design by using the two-phase filtering approach with $R_{\min }=7 \Delta$. a First layer conductivity distribution, $z=1.27 \mathrm{~mm}$. b Second layer conductivity distribution, $z=2.54 \mathrm{~mm}$. $\mathbf{c}$ The scattering parameters of the transition 


\section{Conclusion}

The filtering of the design variables is essential to counteract the self penalization of the optimization problem and to avoid designs with poor performance. The simplest use of filtering, that is, a linear filter combined with a continuation approach over the filter radius, makes it possible to combat the self penalization issue; however, undesirable small features sometimes appear in the final design. To control the size of the small features in the design, we propose a twophase filtering approach, based on a cascade of $f W$-mean filters. The numerical experiments, cross-verified with a commercial solver, indicate that the proposed filtering approach makes it possible to impose minimum size control with minor impact on the performance of the designs.

Acknowledgments This work is financially supported by the Swedish Foundation for Strategic Research (No. AM13-0029), the Swedish Research Council (No. 621-3706), and by the Swedish strategic research program eSSENCE. The computations were performed on resources provided by the Swedish National Infrastructure for Computing (SNIC) at LUNARC and HPC2N centers.

Open Access This article is distributed under the terms of the Creative Commons Attribution 4.0 International License (http:// creativecommons.org/licenses/by/4.0/), which permits unrestricted use, distribution, and reproduction in any medium, provided you give appropriate credit to the original author(s) and the source, provide a link to the Creative Commons license, and indicate if changes were made.

\section{References}

Aage N (2011) Topology optimization of radio frequency and microwave structures. PhD thesis, Technical University of Denmark

Aage N, Egede Johansen V (2017) Topology optimization of microwave waveguide filters. Int J Numer Methods Eng 112(3):283300. https://doi.org/10.1002/nme.5551. ISSN 1097-0207

Akgun O, Tretyakov OA (2015) Solution to the Klein-Gordon equation for the study of time-domain waveguide fields and accompanying energetic processes. IET Microwaves Antennas Propag 9(12):1337-1344. https://doi.org/10.1049/iet-map.2014.0512

Balanis CA (2005) Antenna Theory: Analysis and Design, 3rd edn. Wiley-Interscience, New Jersey

Bang JH, Ahn BC (2014) Coaxial-to-circular waveguide transition with broadband mode-free operation. Electron Lett 50(20):14531454. https://doi.org/10.1049/el.2014.2667

Bendsøe MP, Sigmund O (2003) Topology optimization. theory, methods, and applications. Springer, Berlin

Bialkowski ME, Schwering FK, Morgan MA (2000) On the link between top-hat monopole antennas, disk-resonator diode mounts, and coaxial-to-waveguide transitions [and reply]. IEEE Trans Antennas Propag 48(6):1011-1014. https://doi.org/10.1109/8.86 5244

Bourdin B (2001) Filters in topology optimization. Int J Numer Methods Eng 50(9):2143-2158. https://doi.org/10.1002/nme.116

Bruns TE, Tortorelli DA (2001) Topology optimization of non-linear elastic structures and compliant mechanisms. Comput Methods Appl Mech Eng 190(26-27):3443-3459. https://doi.org/10.1016/ S0045-7825(00)00278-4
Chang CW, Chen Y, Qian J (1997) Nondestructive determination of electromagnetic parameters of dielectric materials at X-band frequencies using a waveguide probe system. IEEE Trans Instrum Meas 46(5):1084-1092. https://doi.org/10.1109/19.676717

Deshpande M, Das B, Sanyal G (1979) Analysis of an end launcher for an X-band rectangular waveguide. IEEE Trans Microw Theory Techn 27(8):731-735. https://doi.org/10.1109/TMTT.112 9715

Diaz AR, Sigmund O (2010) A topology optimization method for design of negative permeability metamaterials. Struct Multidiscip Optim 41(2):163-177. https://doi.org/10.1007/s00158-0090416-y

Erentok A, Sigmund O (2011) Topology optimization of subwavelength antennas. IEEE Trans Antennas Propag 59(1):58-69. https://doi.org/10.1109/TAP.2010.2090451

Guest JK, Prévost JH, Belytschko T (2004) Achieving minimum length scale in topology optimization using nodal design variables and projection functions. Int J Numer Methods Eng 61(2):238254. https://doi.org/10.1002/nme.1064

Guest JK, Asadpoure A, Ha SH (2011) Eliminating beta-continuation from heaviside projection and density filter algorithms. Struct Multidiscip Optim 44(4):443-453. https://doi.org/10.1007/s00158011-0676-1

Hägg L, Wadbro E (2017) Nonlinear filters in topology optimization: existence of solutions and efficient implementation for minimum compliance problems. Struct Multidiscip Optim 55(3):1017-1028. https://doi.org/10.1007/s00158-016-1553-8

Haralick RM, Sternberg SR, Zhuang X (1987) Image analysis using mathematical morphology. IEEE Trans Pattern Anal Mach Intell 9(4):532-550. https://doi.org/10.1109/TPAMI.1987.4767941

Hassan E, Wadbro E, Berggren M (2014a) Patch and ground plane design of microstrip antennas by material distribution topology optimization. Progress Electromagn Res B 59:89-102. https://doi.org/10.2528/PIERB14030605

Hassan E, Wadbro E, Berggren M (2014b) Topology optimization of metallic antennas. IEEE Trans Antennas Propag 63(5):2488-2500. https://doi.org/10.1109/TAP.2014.2309112

Hassan E, Noreland D, Augustine R, Wadbro E, Berggren M (2015a) Topology optimization of planar antennas for wideband nearfield coupling. IEEE Trans Antennas Propag 63(9):4208-4213. https://doi.org/10.1109/TAP.2015.2449894

Hassan E, Wadbro E, Berggren M (2015b) Time-domain sensitivity analysis for conductivity distribution in Maxwell's equations. Department of Computing Science, Umeå University, Technical Report UMINF 1506

Hassan E, Noreland D, Wadbro E, Berggren M (2017) Topology optimisation of wideband coaxial-to-waveguide transitions. Sci Rep 7(3):45,110. https://doi.org/10.1038/srep45110

Heijmans H (1995) Mathematical morphology: A modern approach in image processing based on algebra and geometry. SIAM Rev 37(1):1-36. https://doi.org/10.1137/1037001

Keam R, Williamson A (1994) Broadband design of coaxial line/rectangular waveguide probe transition. IEE Proc Microw Antennas Propag 141(1):53-58. https://doi.org/10.1049/ip-map: 19949798

Kiziltas G, Kikuchi N, Volakis JL, Halloran J (2004) Topology optimization of dielectric substrates for filters and antennas using SIMP. Arch Comput Methods Eng 11(4):355-388. https://doi.org/10.1007/BF02736229

Kristensson G (1993) Transient electromagnetic wave propagation in waveguides. Technical Report LUTEDX/(TEAT-7026)/1-24/

Lathi BP (1998) Modern digital and analog communication systems, 3rd edn. Oxford University Press, Oxford

Nomura T, Sato K, Taguchi K, Kashiwa T, Nishiwaki S (2007) Structural topology optimization for the design of broadband 
dielectric resonator antennas using the finite difference time domain technique. Int J Numer Methods Eng 71:1261-1296. https://doi.org/10.1002/nme.1974

Nomura T, Ohkado M, Schmalenberg P, Lee J, Ahmed O, Bakr M (2013) Topology optimization method for microstrips using boundary condition representation and adjoint analysis. In: 2013 European Microwave Conference, pp 632-635

Otomori M, Yamada T, Izui K, Nishiwaki S, Andkjr J (2012) A topology optimization method based on the level set method for the design of negative permeability dielectric metamaterials. Comput Methods Appl Mech Eng 237-240:192-211. https://doi.org/10.1016/j.cma.2012.04.022

Pellegrini A, Monorchio A, Manara G, Mittra R (2014) A hybrid mode matching-finite element method and spectral decomposition approach for the analysis of large finite phased arrays of waveguides. IEEE Trans Antennas Propag 62(5):2553-2561. https://doi.org/10.1109/TAP.2014.2303826

Pozar D (2012) Microwave engineering, 4th edn. Wiley, New Jersey

Schevenels M, Sigmund O (2016) On the implementation and effectiveness of morphological close-open and open-close filters for topology optimization. Struct Multidiscip Optim 54(1):15-21. https://doi.org/10.1007/s00158-015-1393-y

Sigmund O (1994) Design of material structures using topology optimization. PhD thesis, Technical University of Denmark

Sigmund O (2007) Morphology-based black and white filters for topology optimization. Struct Multidiscip Optim 33(4-5):401424. https://doi.org/10.1007/s00158-006-0087-x
Simeoni M, Coman C, Lager I (2006) Patch end-launchers-a family of compact colinear coaxial-to-rectangular waveguide transitions. IEEE Trans Microw Theory Techn 54(4):1503-1511. https://doi. org/10.1109/TMTT.2006.871923

Svanberg K (2002) A class of globally convergent optimization methods based on conservative convex separable approximations. SIAM J Optim 12(2):555-573. https://doi.org/10.1137/S1052623 499362822

Svanberg K, Svärd H (2013) Density filters for topology optimization based on the pythagorean means. Struct Multidiscip Optim 48(5):859-875. https://doi.org/10.1007/s00158-013-0938-1

Taflove A, Hagness S (2005) Computational Electrodynamics: The Finite-Difference Time-Domain Method, 3rd edn. Artech House, USA

Tako N, Levine E, Kabilo G, Matzner H (2014) Investigation of thick coax-to-waveguide transitions. In: EuCAP 2014, pp 908-911. https://doi.org/10.1109/EuCAP.2014.6901909

Wadbro E, Hägg L (2015) On quasi-arithmetic mean based filters and their fast evaluation for large-scale topology optimization. Struct Multidiscip Optim 52(5):879-888. https://doi.org/10.1007/s00158015-1273-5

Yi W, Li E, Guo G, Nie R (2011) An X-band coaxial-torectangular waveguide transition. In: ICMTCE 2011, pp 129-131. https://doi.org/10.1109/ICMTCE.2011.5915181

Zhou S, Li W, Li Q (2010) Level-set based topology optimization for electromagnetic dipole antenna design. J Comput Phys 229(19):6915-6930. https://doi.org/10.1016/j.jcp.2010.05.030 